\title{
Optimización de los Niveles de los Agentes Oxidante/Reductor en la Panificación de Harina de Trigo
}

\author{
Nibia M. Alasino, Elena E. Aringoli y Hugo D. Sánchez* \\ Instituto de Tecnología de Alimentos, Facultad de Ingeniería Química, Universidad Nacional del \\ Litoral, $1^{\circ}$ de Mayo 3250-Santa Fe-Argentina (e-mail: hsanchez@fiq.unl.edu.ar) \\ * Autor a quien debe ser dirigida la correspondencia
}

Recibido Feb. 28, 2011; Aceptado May. 02, 2011; Versión Final recibida May. 12, 2011

\begin{abstract}
Resumen
El objetivo de este trabajo fue optimizar los niveles del par oxidante/reductor para mejorar el comportamiento tecnológico de harinas. Para este fin se utilizaron las combinaciones: azodicarbonamida-bisulfito de sodio y ácido ascórbico-bisulfito de sodio. Se realizaron ensayos de panificación, determinando consistencia de la masa al final del amasado, volumen específico de pan y asignación de puntaje al pan y a la textura de miga a través de evaluación sensorial. Los resultados indicaron que el uso simultáneo de azodicarbonamida y un bajo nivel de bisulfito de sodio producen un ablandamiento de la masa con disminución de volumen específico del pan. Esto no ocurre, sin embargo, cuando el agente reductor se agrega en niveles cercanos a 100ppm. El uso simultáneo de ácido ascórbico y bisulfito de sodio, en niveles intermedios, produce un efecto benéfico muy importante por lo que su uso resulta altamente recomendable.
\end{abstract} sodio

\section{Optimization of the Levels Oxidant/Reducer Agents in the Baking of Wheat Flour}

\begin{abstract}
The objective of this work was to optimize the levels of oxidant/reducer substances to improve the technological behaviour of flours. Combinations of azodicarbonamide and ascorbic acid with a reducing agent as sodium bisulfite were used. Experiences in bread-making were made determining the dough consistency at the end of mixing and the specific volume of bread. Also, the bread score and the texture score were obtained trough sensorial evaluation. The results indicated that in the case of simultaneous use of azodicarbonamide and low levels of sodium bisulfite produce a softening of the dough with a decrease in specific volume of bread. This does not occur when the reducing agent is added at levels close to $100 \mathrm{ppm}$. The simultaneous use of ascorbic acid and sodium bisulfite, at intermediate levels, produces a very significant beneficial effect, so its use is highly recommended.
\end{abstract}

Keywords: baking, oxidant-reducer, ascorbic acid,azodicarbonamide, sodium bisulfite 


\section{INTRODUCCIÓN}

El componente más importante de la harina en relación con su uso en panificación es la proteína ya que las propiedades de la masa están relacionadas al comportamiento viscoelástico del gluten. Las proteínas formadoras del gluten como las gliadinas y las gluteninas se encuentran principalmente entrecruzadas por puentes disulfuro. Las gluteninas consisten en una mezcla heterogénea de polímeros, existiendo dos clases principales de subunidades, la subunidad de alto peso molecular (HMW-GS, High molecular weight glutenin subunits) y la subunidad de bajo peso molecular (LMG-GS, low molecular weight glutenin subunits)(Veraverbeke et al., 2000; Goesaert et al., 2005)). La subunidad HMW juega un rol sumamente importante en la formación del gluten y numerosos estudios han demostrado la importancia de la estructura y cantidad de estas subunidades de HMW para la reología de la masa y para la calidad de la panificación de diferentes trigos (Antes y Wieser, 2001).

Para obtener propiedades satisfactorias en el proceso de producción de pan, es esencial un apropiado desarrollo del gluten, su punto óptimo de desarrollo es difícil alcanzar, ya que varía con el tipo de harina usada y además es influenciada por factores adicionales como temperatura de masa, velocidad de amasado, absorción de agua, tipo y cantidad de oxidantes, enzimas suplementarias, etc. (Pyler, 1973a). Las cadenas que forman el gluten interaccionan entre sí o con sustancias (lípidos y glúcidos) formando puentes que crean una red más o menos densa. La densidad y solidez de estos puentes y la elasticidad de la red van a determinar la capacidad que tiene la masa de fermentar o de retener el anhídrido carbónico. Las uniones que se logran son de dos tipos, las uniones peptídicas como las que se dan entre los aminoácidos y las uniones disulfuro obtenidas de la unión de dos moléculas de cisteina por deshidrogenación. Mientras que los enlaces peptídicos dependen del pH la formación de los puentes disulfuros dependen del potencial de óxido-reducción y en particular de la presencia de oxidantes (Quaglia, 1991; Lagrain et al., 2006). Es así como las propiedades reológicas de la masa y su estructura tridimensional serán dependientes de las interacciones sulfhidrilo (SH) y disulfuro (SS) (Nakamura y Kurata, 1997a). La remoción de grupos SH a través de la acción de oxidantes químicos como el iodato o el bromato de potasio, o por la reacción de agentes bloqueantes como $\mathrm{N}$-etilamida, generalmente mejoran la calidad de la masa, presumiblemente evitando la rotura de los puentes SS y de esa manera mejorando las caracerísticas del pan (Yamada y Preston, 1992, 1994). Los agentes oxidantes y reductores afectan las propiedades mecánicas de la masa y del gluten muy especialmente a través de la modificación del estado redox de los grupos sulfhidrilos (SH) y puentes disulfuro (SS) (Larré et al., 2000).

Cuando la masa moderadamente madurada es tratada con pequeños pocentajes de agentes oxidantes, se vuelve más elástica, más compacta y menos pegajosa que la masa no aditivada. El pan resultante tiene un buen volumen y su textura es suave y aterciopelada. En cambio, cuando se agrega demasiado agente oxidante, resulta una sobreoxidación y el pan hecho en tales condiciones resulta con pequeño volumen, su miga exhibe un textura rígida y con pequeños alveolos (Pyler, 1973b). Cuando se agrega ácido ascórbico como aditivo, éste reacciona sobre los grupos sulfidrilos de una harina previo haber recibido la acción de la ácidodehidroascórbicoreductasa presente en la harina con la pérdida de dos átomos de hidrógeno y la formación del ácido dehidroascórbico que es el agente que actúa como oxidante (Lagrain et al., 2008; Maforimbo et al., 2007; Lu y Seib, 1998; Kaid et al., 1997; Every et al., 1996). Otro oxidante difundido a partir de 1972 es la azodicarbonamida (ADA) que al igual que el ácido ascórbico tiene la propiedad de convertir los grupos sulfidrilos (SH) en disulfuros (SS) dejando residuos no agresivos al resto del sistema que constituye la masa (Fitchett y Frazier, 1986).

Por otra parte, aditivos como las endopeptidasas y los agentes reductores (bisulfito de sodio y Lcisteína) producen una acción de ruptura de enlaces; en el primer caso es sobre las uniones peptídicas internas de las proteínas del gluten, y en el segundo es sobre las uniones disulfuro; en ambos casos se produce un aumento de la extensibilidad del gluten pero por vías de acción totalmente diferentes (Sroan y Kaur, 2004; Lambert y Kokini, 2001; Osella et al., 1995; Bloksma y Bushuk, 1988; Fitchett y Frazier, 1986; Linko, 1985). El objetivo de este trabajo fue optimizar los niveles de combinaciones oxidante-reductor para mejorar el comportamiento tecnológico de 
harinas. Para este fin se utilizaron combinaciones seleccionadas, según diseño experimental, de azodicarbonamida-bisulfito de sodio y de ácido ascórbico-bisulfito de sodio.

\section{MATERIALES Y MÉTODOS}

\section{Materias primas}

La harina de trigo, apta para la panificación en la industria argentina, fue provista por Molinos Matilde Santa Fe (Argentina), con las siguientes características: 11,7\% humedad, 11,0\% proteina (\% Nx5,7), 1,62\% grasa, $0,52 \%$ cenizas. Sus propiedades físicas evaluadas en farinógrafo Brabender fueron: absorción de agua 58,1\%, desarrollo 2,3min, estabilidad 4,1min y ablandamiento 100 unidades Brabender(BU); y sus propiedades físicas evaluadas en el alveógrafo Chopin fueron: energía de deformación $(W)=205$ Joules $\times 10^{-4}$ y relación elasticidad/extensibilidad $(P / L)=1,10$. La materia grasa utilizada fue oleomargarina marca Optima (punto de fusión $36^{\circ} \mathrm{C}$ ), de Molinos Rio de la Plata S.A., Buenos Aires. Los aditivos usados como agentes oxidantes fueron la azodicarbonamida (ADA) y el acido ascórbico (Ac Asc) y como agente reductor el bisulfito de sodio (Bis de $\mathrm{Na}$ ). Estos aditivos se utilizaron combinados de a pares: oxidante-reductor.

\section{Propiedades físicas de la masa}

Estas propiedades evaluadas en la masa fueron determinadas a través del farinógrafo Brabender y del alveógrafo Chopin, basados en los métodos 54-21 y 54-30 (AACC 1994).

\section{Ensayos de panificación}

Los ingredientes (300 $\mathrm{g}$ de harina de trigo, $15 \mathrm{~g}$ de levadura, $6 \mathrm{~g}$ de sal, $18 \mathrm{~g}$ de azúcar, $9 \mathrm{~g}$ de oleomargarina y $6 \mathrm{~g}$ de leche descremada en polvo) se mezclaron durante un minuto en el Farinógrafo Brabender. Se agregó el agua con los aditivos a una temperatura que permitiera obtener una masa con $24-26^{\circ} \mathrm{C}$ al final del amasado. Despues de $10 \mathrm{~min}$ de amasado a $60 \mathrm{rpm}$, se midió la consistencia (UB) de la masa y se llevó a fermentar en cámara a $27^{\circ} \mathrm{C}$ y $80 \%$ de humedad relativa, controlando dicha etapa con el medidor de empuje. Este aparato consiste en un recipiente cilindrico de vidrio (altura $75 \mathrm{~mm}$, diametro interior $45 \mathrm{~mm}$ ) con un piston de plástico liviano que se eleva durante la fermentación. En todos los casos el agua agregada fue de 58,1\% respecto a la harina, este valor corresponde a la absorción de agua en farinógrafo por parte de la harina sin aditivar. La primera fermentación se da por terminada cuando se duplica el volumen en el medidor de empuje (de 12,5mm a 25,0mm en aproximadamente $40 \mathrm{~min}$ ). Luego dos trozos de masa de $250 \mathrm{~g}$ son laminados, enrollados y colocados en moldes para una segunda fermentación. Esta última concluye cuando se cuadruplica el volumen de masa, el medidor de empuje se desplaza de $15,0 \mathrm{~mm}$ a $60,0 \mathrm{~mm}$ en aproximadamente $75 \mathrm{~min}$. Los moldes metálicos utilizados son de una altura de $55 \mathrm{~mm}$, con una superficie en la base de $70 \mathrm{~mm} \times 175 \mathrm{~mm}$ y en la parte superior de $90 \mathrm{~mm} \times 180 \mathrm{~mm}$. Las moldes con la masa ya fermentada se llevó a cocción a $210^{\circ} \mathrm{C}$ durante $25 \mathrm{~min}$ en horno eléctrico (Ojalvo S.A., Santa Fe, Argentina). El volumen específico del pan (ml/g) se determinó 60 min después de la cocción por el sistema de desplazamiento de semillas. Luego los panes obtenidos fueron evaluados con asignación de puntaje a sus características físicas con una base de comparación. Cinco Expertos asignaron los puntajes a características individuales del pan comparandolas con un estándar hipotético. De acuerdo a Pyler (1973) para evaluación de un pan blanco estándar y con modificaciones según Sánchez et al. (1983), un esquema típico de evaluación presenta los siguientes puntajes máximos: Volume, 15 (volumen específico de $5 \mathrm{ml} / \mathrm{g}$ corresponde al valor máximo); corteza, 15 (color y espesor); textura de miga, 15 (elasticidad y pegajosidad); color de miga, 10 (blanco-crema es el óptimo); estructura de miga, 10 (tamaño y forma de alveolos); aroma, 15 (aroma a pan fresco); y sabor, 20 (flavor y sensación en la boca). El puntaje del pan se califica de la siguiente manera (Tosi et al., 2002): Excelente (90-100), muy bueno (80-89), bueno (70-79), aceptable (60-69), pobre (50-59), muy pobre (40-49), extremadamente pobre (30-39). Cuando la textura de la miga se evalúa individualmente su puntuación se efectúa en una escala 0-10. 


\section{Diseño experimental}

Se evaluaron cuatro respuestas para las siguientes combinaciones oxidante-reductor: ADA-Bis de $\mathrm{Na}\left(Y_{i}\right)$ y Ac Asc-Bis de $\mathrm{Na}\left(Y_{i}^{\prime}\right)$. Tales respuestas fueron: Consistencia de la masa al final del amasado $\left(\mathrm{Y}_{1}, \mathrm{Y}_{1}{ }^{\prime}\right)$, volumen especifico del pan $\left(\mathrm{Y}_{2}, \mathrm{Y}_{2}{ }^{\prime}\right)$, puntaje del pan $\left(\mathrm{Y}_{3}, \mathrm{Y}_{3}{ }^{\prime}\right)$, puntaje de la textura de miga $\left(Y_{4}, Y_{4}{ }^{\prime}\right)$. Las variables seleccionadas fueron: oxidante $\left(X_{1}\right)$ (100 a 200ppm) y reductor $\left(X_{2}\right)(50$ a $100 \mathrm{ppm})$ (punto central: $\left.X_{1}=150 X_{2}=75 \mathrm{ppm}\right)$. las variables a ser optimizadas se codificaron en cinco niveles (Tabla 1). La selección de los niveles extremos fue llevada a cabo en estudios previos. Se realizó un diseño central compuesto, como se muestra en Tabla 2, adaptado a un modelo de segundo orden. Se incorporaron los puntos en estrella al diseño factorial a efectos de estimar la curvatura del modelo (Montgomery 1991). Se efectuaron cuatro repeticiones en el centro del diseño para determinar el error puro en la suma de cuadrados.

Tabla 1 : Diseño Central Compuesto-Variables y Niveles

\begin{tabular}{ccccccc}
\hline & & \multicolumn{5}{c}{ Niveles de las variables codificadas } \\
\hline Variable & Símbolo & -1.41421 & -1 & 0 & 1 & 1.41421 \\
\hline Oxidante $(\mathrm{ppm})$ & $\mathrm{X}_{1}$ & 79 & 100 & 150 & 200 & 221 \\
Reductor $(\mathrm{ppm})$ & $\mathrm{X}_{2}$ & 40 & 50 & 75 & 100 & 110 \\
\hline
\end{tabular}

Tabla 2: Diseño Central Compuesto-Combinaciones y Respuestas. $Y_{1}, Y^{\prime}{ }_{1}=$ consistencia de la masa al final del amasado; $Y_{2}, Y_{2}^{\prime}=$ volumen específico del pan; $Y_{3}, Y^{\prime}{ }_{3}=$ puntaje del pan $(\max$. $100) ; Y_{4}, Y^{\prime}{ }_{4}=$ puntaje de la textura de miga (max.15).

\begin{tabular}{ccccccccccc}
\hline & \multicolumn{2}{c}{$\begin{array}{c}\text { Variables } \\
\text { codificadas }\end{array}$} & \multicolumn{7}{c}{ ADA-Bis de Na } \\
\cline { 3 - 10 } Exp. & $\mathrm{X}_{1}$ & $\mathrm{X}_{2}$ & $\mathrm{Y}_{1}$ & $\mathrm{Y}_{2}$ & $\mathrm{Y}_{3}$ & $\mathrm{Y}_{4}$ & $\mathrm{Y}_{1}^{\prime}$ & $\mathrm{Y}_{2}^{\prime}$ & $\mathrm{Y}_{3}^{\prime}$ & $\mathrm{Y}_{4}^{\prime}$ \\
\hline 1 & 1 & -1 & 500 & 4.48 & 83.4 & 12.5 & 470 & 4.34 & 84.3 & 12.8 \\
2 & 0 & -1.414 & 560 & 4.19 & 84.9 & 12.8 & 480 & 4.24 & 81.7 & 13.5 \\
3 & 0 & 0 & 550 & 4.70 & 87.6 & 13.0 & 470 & 4.60 & 87.0 & 12.8 \\
4 & 0 & 0 & 530 & 4.63 & 86.4 & 13.0 & 560 & 4.76 & 89.0 & 13.8 \\
5 & 1.414 & 0 & 500 & 4.68 & 87.3 & 13.1 & 500 & 4.71 & 89.8 & 13.5 \\
6 & 0 & 1.414 & 620 & 5.00 & 91.0 & 13.5 & 540 & 4.94 & 93.5 & 13.8 \\
7 & -1.414 & 0 & 570 & 4.94 & 89.8 & 13.5 & 570 & 4.74 & 89.2 & 12.0 \\
8 & -1 & -1 & 560 & 4.82 & 88.0 & 13.5 & 580 & 4.92 & 88.8 & 13.5 \\
9 & 1 & -1 & 560 & 4.54 & 88.6 & 13.3 & 450 & 4.16 & 83.0 & 12.0 \\
10 & 1 & 1 & 600 & 4.90 & 89.5 & 13.5 & 470 & 4.61 & 88.8 & 12.8 \\
11 & 1 & -1 & 480 & 4.46 & 82.7 & 12.8 & 470 & 4.38 & 84.6 & 12.0 \\
12 & 0 & -1.414 & 550 & 4.22 & 83.0 & 12.8 & 490 & 4.27 & 81.8 & 12.0 \\
13 & 0 & 0 & 530 & 4.75 & 89.30 & 13.5 & 590 & 4.66 & 88.3 & 12.8 \\
14 & 0 & 0 & 530 & 4.63 & 86.4 & 12.9 & 490 & 4.88 & 90.0 & 13.5 \\
15 & 1.414 & 0 & 520 & 4.64 & 83.4 & 12.5 & 520 & 4.65 & 89.0 & 13.50 \\
16 & 0 & 1.414 & 630 & 4.90 & 91.5 & 14.0 & 540 & 4.88 & 88.2 & 13.5 \\
17 & -1.414 & 0 & 580 & 4.90 & 91.2 & 14.0 & 560 & 4.65 & 89.0 & 12.8 \\
18 & -1 & -1 & 580 & 4.80 & 87.4 & 13.5 & 590 & 4.90 & 93.4 & 13.8 \\
19 & -1 & 1 & 570 & 4.50 & 87.5 & 13.5 & 460 & 4.21 & 83.4 & 11.8 \\
20 & 1 & 1 & 600 & 5.00 & 91.5 & 14.0 & 480 & 4.63 & 88.8 & 13.5 \\
\hline & & & & & & & & &
\end{tabular}




\section{Análisis estadístico}

Se utilizó el software Statgraphics para lograr el modelo de segundo orden y generar las superficies de respuesta. El modelo propuesto para cada respuesta esta dado por la ecuación (1).

$Y=b_{0}+b_{1} X_{1}+b_{2} X_{2}+b_{11} X_{1}^{2}+b_{22} X_{2}^{2}+b_{12} X_{1} X_{2}$

donde $b_{0}$ es el valor de la respuesta en el punto central $(0,0) ; b_{1}$ y $b_{2}$ son los términos de regresión lineales; $b_{11}$ y $b_{22}$ son los términos de regresión cuadráticos; y $b_{12}$ es el término de regresión del producto cruzado (Montgomery 1991).

\section{RESULTADOS Y DISCUSIÓN}

El análisis de varianza (Tabla 3 ) muestra los cambios estadísticamente significativos que se producen en algunas respuestas con la adición de oxidante $\left(X_{1}\right)$ y reductor $\left(X_{2}\right)$. El oxidante ADA produce un efecto significativo sobre todas las respuestas excepto sobre el volumen específico, mientras que el oxidante Ac Asc lo produce sobre todas las respuestas excepto sobre el puntaje del pan. En cuanto al agente reductor Bis de $\mathrm{Na}$ se puede apreciar su efecto significativo sobre la mayoría de las respuestas. Un hecho destacable es la importante influencia de la acción combinada oxidante-reductor sobre el volumen específico tanto para el caso en que se utiliza ADA-Bis de Na como para el caso en que se utiliza Ac Asc-Bis de Na. Respecto a la falta de ajuste, todos los valores de $p$ son mayores que 0,05 lo que indica aceptar el modelo utilizado como apropiado en todos los casos.

Tabla 3: Resultados del Análisis de Varianza. $Y_{1}, Y^{\prime}{ }_{1}=$ consistencia de la masa al final del amasado; $Y_{2}, Y_{2}^{\prime}=$ volumen específico del pan; $Y_{3}, Y^{\prime}{ }_{3}=$ puntaje del pan $(\max .100) ; Y_{4}, Y_{4}^{\prime}=$ puntaje de la textura de miga (max. 15). Significación estadística: * $P<0,05$

Valor $\mathrm{F}$

\begin{tabular}{cccccccccc}
\hline & \multicolumn{1}{c}{ ADA-Bis de Na } & \multicolumn{5}{c}{ Ac Asc-Bis de Na } \\
\hline Variable & $\begin{array}{c}\text { Grados de } \\
\text { libertad }\end{array}$ & $\mathrm{Y}_{1}$ & $\mathrm{Y}_{2}$ & $\mathrm{Y}_{3}$ & $\mathrm{Y}_{4}$ & $\mathrm{Y}_{1}^{\prime}$ & $\mathrm{Y}^{\prime}{ }_{2}$ & $\mathrm{Y}^{\prime}{ }_{3}$ & $\mathrm{Y}_{4}^{\prime}$ \\
\hline $\mathrm{X}_{1}$ & 1 & $46.9^{*}$ & 4.0 & $11.2^{*}$ & $16.4^{*}$ & $14.3^{*}$ & $1992.0^{*}$ & 7.0 & $45.3^{*}$ \\
$\mathrm{X}_{2}$ & 1 & $104.0^{*}$ & $80.1^{*}$ & $33.4^{*}$ & $17.3^{*}$ & $12.6^{*}$ & $70.0^{*}$ & 0.0 & $49.0^{*}$ \\
$\mathrm{X}_{1}{ }^{2}$ & 1 & 0.14 & 6.2 & 0.1 & 1.0 & $18.8^{*}$ & $2835.0^{*}$ & 5.5 & $38.3^{*}$ \\
$\mathrm{X}_{2}{ }^{2}$ & 1 & $57.1^{*}$ & 4.6 & 0.0 & 1.0 & 6.54 & $776.3^{*}$ & 1.7 & $32.0^{*}$ \\
$\mathrm{X}_{1} \mathrm{X}_{2}$ & 1 & $66.1^{*}$ & $61.4^{*}$ & $12.2^{*}$ & 8.0 & $12.0^{*}$ & $16.0^{*}$ & 0.3 & 2.8 \\
Falta de ajuste & 3 & 1.7 & 4.7 & 0.8 & 0.7 & 0.5 & 109.6 & 0.3 & 5.1 \\
\hline Coeficiente $\mathrm{R}^{2}$ & & 0.933 & 0.752 & 0.825 & 0.783 & 0.885 & 0.804 & 0.718 & 0.717 \\
\hline
\end{tabular}

\section{Consistencia al final del amasado}

La Figura 1 corresponde a la variación de consistencia de la masa al final del amasado en función de los niveles de oxidante y reductor. La Figura $1(A)$ expresa las variaciones ocurridas por el par ADA-Bis de Na y la Figura 1 (B) las producidas por el par Ac Asc-Bis de Na. En la Figura 1 (A) se puede apreciar el efecto opuesto generado por el ADA respecto al Bis de $\mathrm{Na}$, mientras que con el ADA ocurre un descenso pronunciado de la consistencia, con el Bis de $\mathrm{Na}$ se produce un aumento 
de similar magnitud. Esto debe ser interpretado como que el ADA tiene una tendencia a ablandar la masa cuando el Bis de $\mathrm{Na}$ esta presenta en la formulación ya que cuando el ADA se utiliza individualmente tiene un comportamiento diferente, que consiste en producir un aumento de la tenacidad de la masa (Lee et al, 2001). En la Figura 1 (B) se puede ver que con el par Ac Asc-Bis de $\mathrm{Na}$ ocurre un efecto contrario al que se aprecia con el par ADA-Bis de $\mathrm{Na}$. Cuando crece el valor del oxidante Ac Asc tambien crece el valor de la consistencia mientras que ésta disminuye, produciéndose un ablandamiento de la masa, cuando aumenta el nivel de uso de Bis de Na.Según experiencias previas, se recomiendan valores de consistencia de la masa al final del amasado entre 500 y 550 B. Estos valores se logran según Figura 1 (A) con ADA entre 120 y $200 p p m$ y Bis de $\mathrm{Na}$ entre 80 y 100ppm y según Figura 1 (B) con Ac Asc entre 120 y 170ppm y Bis de Na entre 60 y 80 ppm.

(A)

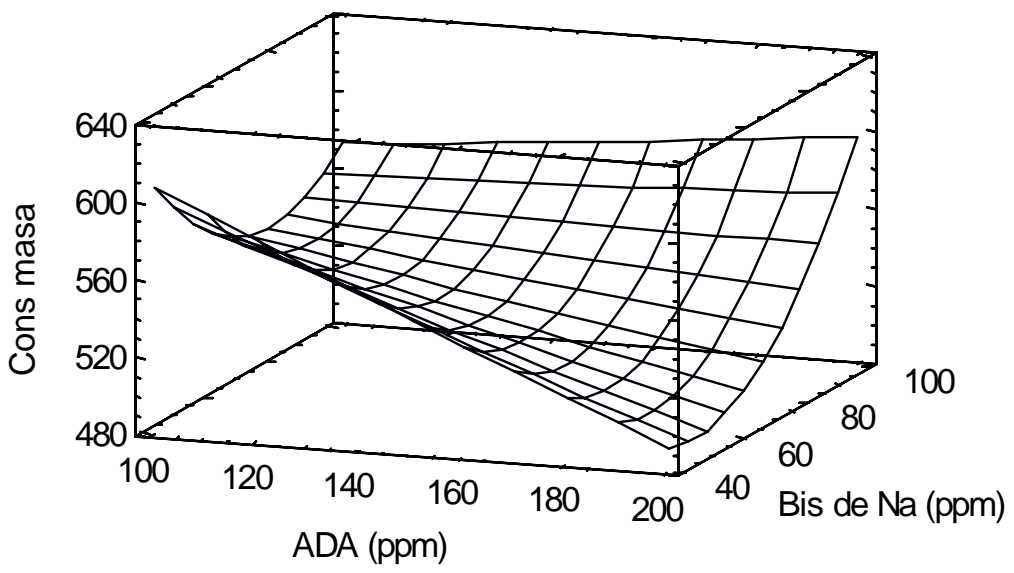

(B)

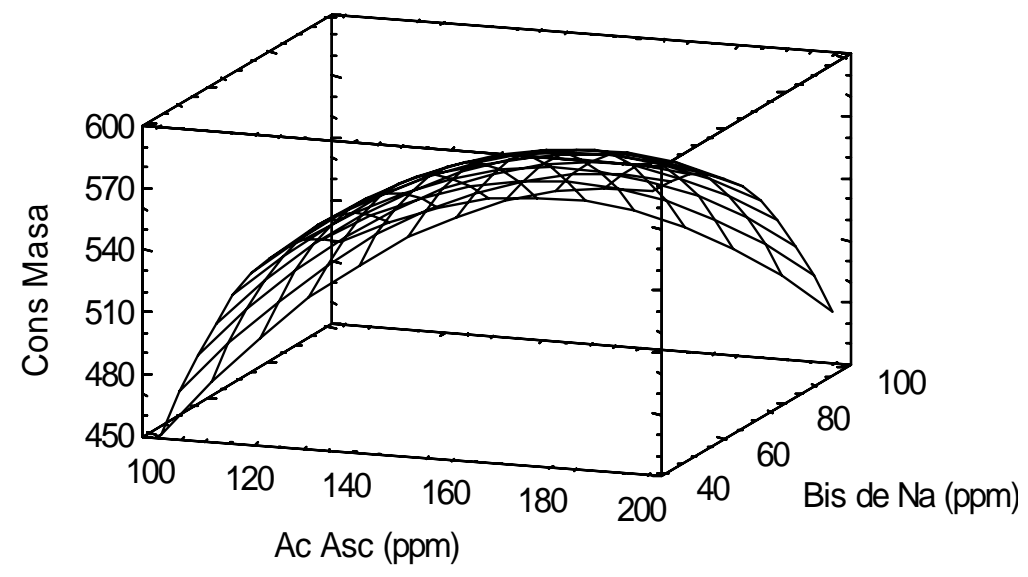

Fig. 1: Superficie de respuesta para $Y_{1}=$ consistencia de la masa al final del amasado (UB). (A) en función de ADA y Bis de Na. (B) en función de Ac Asc y Bis de $\mathrm{Na}$.

\section{Volumen específico del pan}

La Figura 2 corresponde a la variación de volumen específico del pan en función de los niveles de oxidante y reductor. La Figura 2 (A) expresa las variaciones ocurridas por el par ADA-Bis de Na y la Figura 2 (B) las producidas por el par Ac Asc-Bis de Na. En la Figura 2 (A) se puede observar que al ir aumentando el nivel de ADA, en niveles bajos del agente reductor, disminuye el volumen específico y al ir aumentando el nivel de Bis de $\mathrm{Na}$ aumenta el volumen específico. A su vez se debe destacar el crecimiento de volumen específico que produce el ADA cuando en la formulación se cuenta con la presencia de 100 ppm de Bis de $\mathrm{Na}$. El valor máximo de volumen específico se estima en 180-200ppm de ADA con 100ppm de Bis de Na. En la Figura 2 (B) se aprecia que al ir aumentando los niveles de aditivos aumenta el volumen específico hasta un valor determinado a 
partir del cual el volumen específico comienza a disminuir. Esto coincide con el estudio realizado por Yamada \& Preston (1994) quienes encuentran que el agregado de oxidantes por encima de un nivel óptimo produce una disminución del volumen del pan, con lo cual se genera una pérdida de calidad.Se observa que los mayores valores de volumen específico del pan se presentan en un nivel de Ac Asc alrededor de 165ppm y de Bis de Na entre 70 y $90 \mathrm{ppm}$.

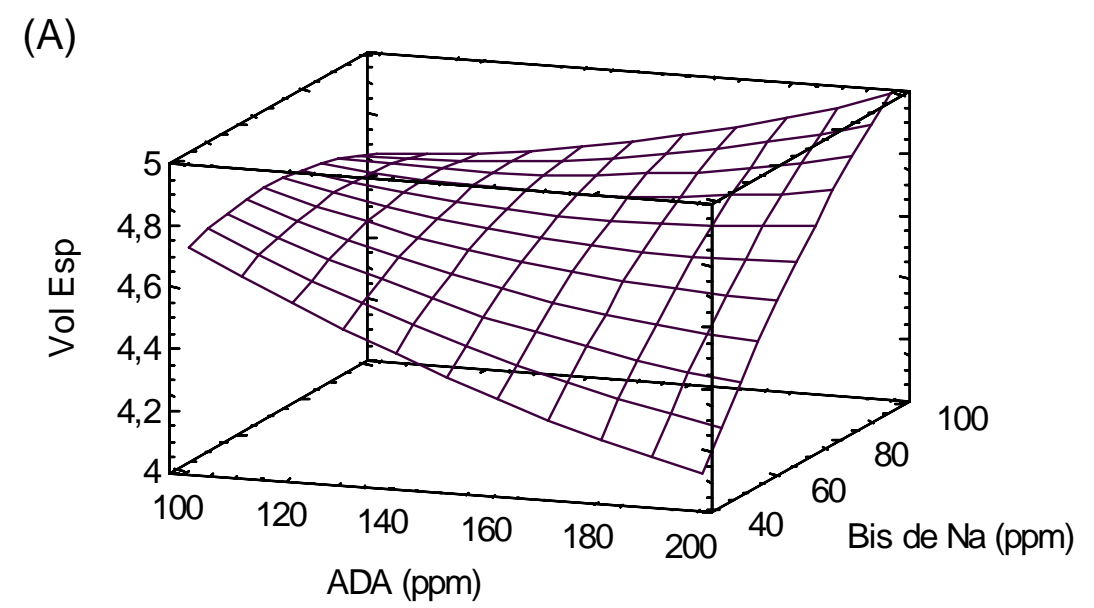

(B)

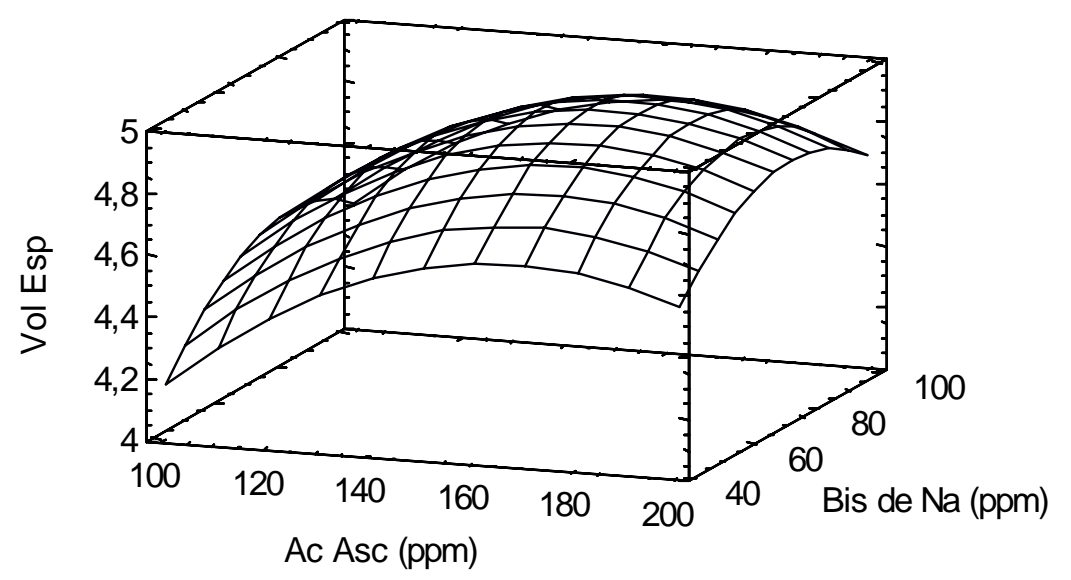

Fig. 2: Superficie de respuesta para $Y_{2}=$ volumen específico del pan $(\mathrm{ml} / \mathrm{g})$.

(A) en función de ADA y Bis de Na. (B) en función de Ac Asc y Bis de $\mathrm{Na}$.

\section{Puntaje del pan}

La Figura 3 corresponde a la variación de puntaje del pan en función de los niveles de oxidante y reductor. La Figura 3 (A) expresa las variaciones ocurridas por el par ADA-Bis de Na y la Figura 3 (B) las producidas por el par Ac Asc-Bis de Na. En la Figura 3 (A) se puede observar que al ir aumentando los niveles de ADA, en niveles bajos del agente reductor, disminuye el puntaje y contrariamente al ir aumentando los niveles de Bis de $\mathrm{Na}$ aumenta dicho puntaje. Los mayores valores se presentan ante un efecto combinado oxidante-reductor, con un nivel de ADA cercano a 200ppm y de Bis de Na de aproximadamente 100ppm. Respecto al par Ac Asc-Bis de Na (Figura 3 (B)) si bien el efecto de los aditivos no es significativo respecto de los atributos sensoriales, se puede admitir que hay una tendencia a aumentar el puntaje otorgado hasta un valor determinado y luego este puntaje disminuye, estando los mayores valores para niveles de Ac Asc cercano a 170ppm y de Bis de Na entre 70-80ppm. 


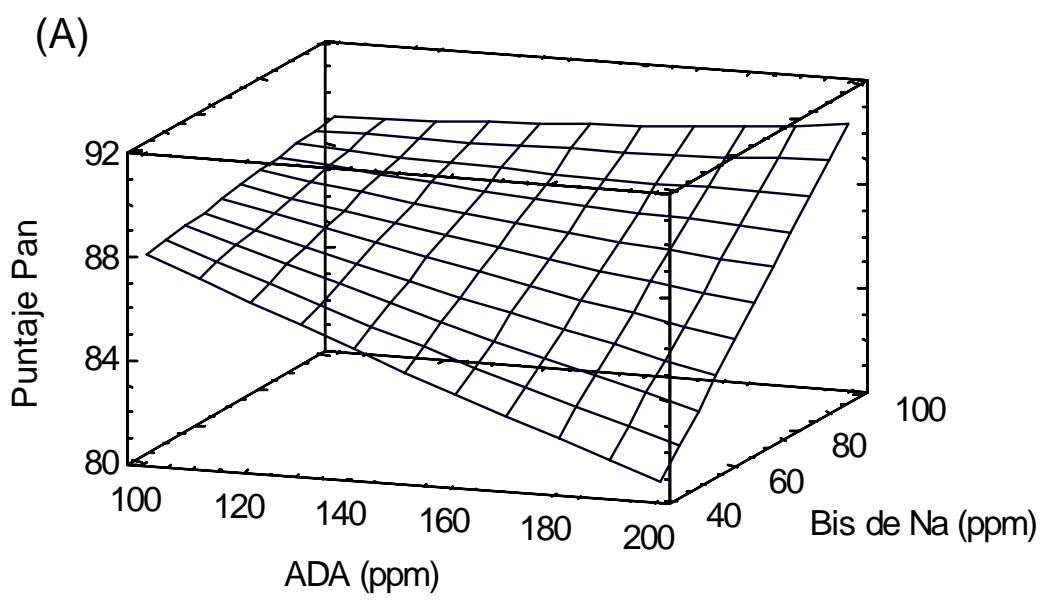

(B)

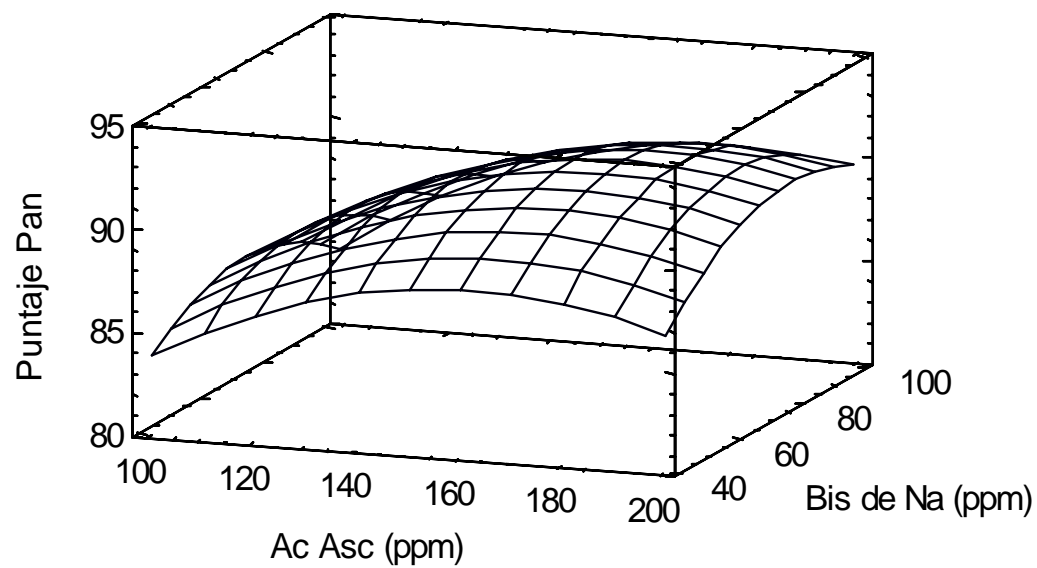

Fig. 3: Superficie de respuesta para $Y_{3}=$ puntaje del pan $(\max 100)$. (A) en función de ADA y Bis de Na. (B) en función de Ac Asc y Bis de Na.

\section{Puntaje de la textura de miga}

La Figura 4 corresponde a la variación de puntaje de la textura de miga en función de los niveles de oxidante y reductor. La Figura 4 (A) expresa las variaciones ocurridas por el par ADA-Bis de $\mathrm{Na}$ y la Figura 4 (B) las producidas por el par Ac Asc-Bis de Na. En ambas figuras se observa que al ir aumentando los niveles de aditivos se produce una variación con similar tendencia que la mostrada en los casos anteriores. El mayor puntaje estimado según Figura $4(A)$ es para niveles de ADA entre 100 y 200ppm y de Bis de Na cercanos a 100ppm. Para el caso de la Figura 4 (B) se observa que se obtienen los mayores valores del puntaje a la textura en la zona correspondiente a Ac Asc 170ppm y Bis de Na entre 70-80ppm.

\section{Valores óptimos}

En Tabla 4 se aprecian los valores de los pares oxidante-reductor que optimizan las siguientes respuestas: Consistencia de la masa al final del amasado, volumen específico del pan y evaluación sensorial por puntaje del pan y por puntaje a la textura de la miga. Además se asignó un punto óptimo global que satisfaga los valores óptimos de cada respuesta y que permita llevar a cabo la verificación del modelo. 
(A)

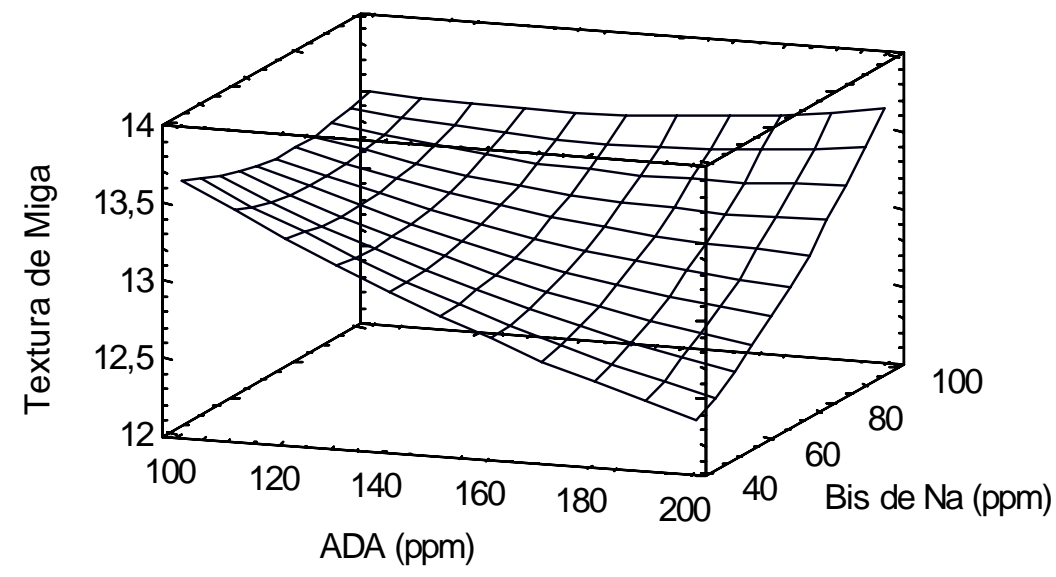

(B)

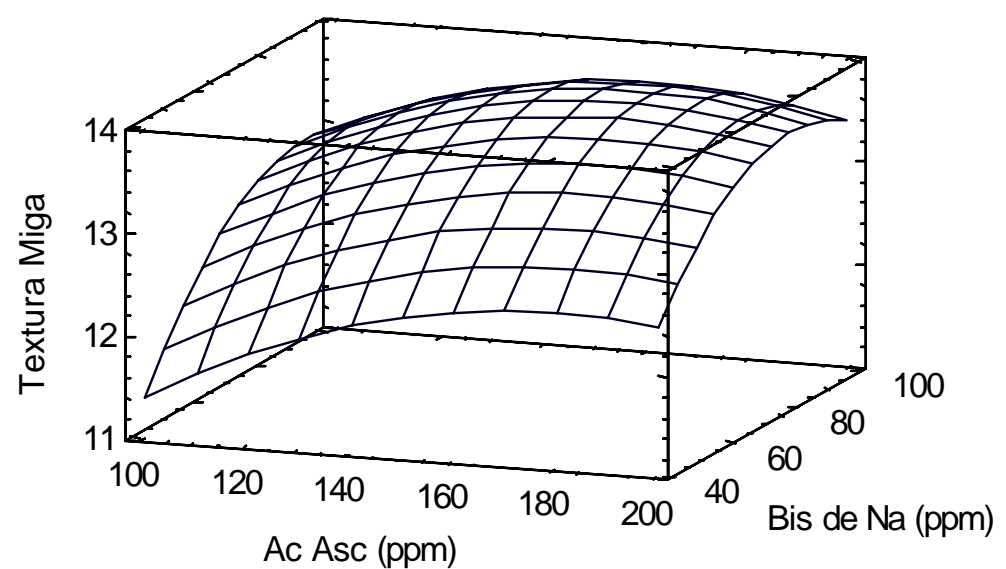

Fig. 4: Superficie de respuesta para $Y_{4}=$ puntaje textura de la miga (max 15). (A) en función de ADA y Bis de Na. (B) en función de Ac Asc y Bis de Na.

\section{Verificación del modelo}

Se llevó a cabo la verificación experimental del modelo para ambos pares oxidante-reductor utilizados. En primer lugar se verificó el par ADA-Bis de $\mathrm{Na}$ con seis experiencias en su punto óptimo global 190ppm-100ppm (Tabla 4). El resultado logrado fue: consistencia de la masa al final del amasado $Y_{1}=580 \mathrm{UB}$, volumen específico del pan $\mathrm{Y}_{2}=4,86 \mathrm{ml} / \mathrm{g}$, puntaje del pan $\mathrm{Y}_{3}=89,7 \mathrm{y}$ puntaje de la textura de miga $Y_{4}=13,5$. En segundo lugar se verificó el par Ac Asc-Bis de $\mathrm{Na}$ también con seis experiencias en su correspondiente punto ótimo global 165ppm-77ppm (Tabla 4). El resultado fue el siguiente: consistencia de la masa al final del amasado $Y^{\prime}{ }_{1}=570 \mathrm{UB}$, volumen específico $Y^{\prime}{ }_{2}=4,74 \mathrm{ml} / \mathrm{g}$, puntaje del pan $\mathrm{Y}_{3}{ }_{3}=90,6$ y puntaje de la textura de miga $\mathrm{Y}_{4}{ }_{4}=$ 14,2. Utilizando la técnica del test de hipótesis (Montgomery 1991), las diferencias entre las respuestas del modelo y de la verificación experimental no son significativas a un nivel del 5\%.

Tabla 4: Valores de los pares Oxidante-Reductor que optimizan las respuestas

\begin{tabular}{lcc|cc}
\hline & ADA & Bis de Na & Ac Asc & Bis de Na \\
\hline Cons. masa & $120-200$ & $80-100$ & $120-170$ & $60-80$ \\
Vol. Esp. & $180-200$ & 100 & 165 & $70-90$ \\
Puntaje pan & 200 & 100 & 170 & $70-80$ \\
Puntaje textura & $100-200$ & 100 & 170 & $70-80$ \\
Punto óptimo global & 190 & 100 & 165 & 77 \\
\hline
\end{tabular}




\section{CONCLUSIONES}

El uso simultáneo de oxidante-reductor produce efectos combinados que en algún caso pueden resultar inesperados. Así para el caso de la azodicarbonamida que siendo utilizada en forma individual produce un efecto de endurecimiento de la masa, cuando se la incorpora en la formulación junto a bajos niveles de reemplazo de un reductor como el bisulfito de sodio, su efecto resulta de manera opuesta, o sea que provoca un ablandamiento de la masa a la vez que una disminución del volumen específico, no así cuando este agente reductor se agrega en niveles cercanos a las 100ppm en donde los resultados mejoran significativamente. En cuanto al uso simultáneo, en niveles intermedios, del ácido ascórbio y el bisulfito de sodio, su combinación produce un efecto benéfico muy importante por lo que su uso resulta también altamente recomendable.

\section{REFERENCIAS}

AACC-American Association of Cereal Chemists, Approved Methods of the AACC. $8^{\text {th }}$ edn, St. Paul, MN. USA (1994).

Antes, S. y H. Wieser, Reoxidation Behavior of Wheat and Rye Glutelin Subunits, Cereal Chemistry, 78, 8-13 (2001).

Bloksma, A.H. y W. Bushuk, Rheology and Chemistry of Dough, In: WHEAT: Chemistry and Technology. Edited by Pomeranz. Y. American Association of Cereal Chemists. St. Paul, Mn. pp 192-193 (1988).

Every, D., M.J. Gilpin y N.J. Larsen, Ascorbate Oxidase levels in Wheat and relationship to Baking Quality, Journal of Cereal Science, 23, 354-359 (1996).

Fitchett, C. y P. Frazier, Action of Oxidants and others Improvers, In: Chemistry and Physics of Baking. Edited by Blanshard J., Frazier, P.y Galliard, T. The Royal Society of Chemistry, Londres, pp.179-198 (1986).

Goesaert, H., Brijs, K., Veraverbeke, W.S., Courtin, C.M., Gebruers, K., Delcour, J.A., Wheat flours constituents: how they impact bread quality, and how to impact their functionality, Trends in food science and technology, 16, 12-30 (2005).

Kaid, N., L. Rakotozafy, J. Potus y J. Nicolas, Studies on the Glutathione-Dehydroascorbate Oxidoreductase from Wheat Flour, Cereal Chemistry, 74, 605-610 (1997).

Lagrain, B., Brijs, K., Delcour, J.A. Impact of redox agents on the physico-chemistry of wheat gluten proteins during hydrothermal treatment, Journal of Cereal Science, 44, 49-53 (2006).

Lagrain, B., Thewissen, B.G., Brijs, K., Delcour, J.A. Mechanism of gliadin-glutenin cross-linking during hydrothermal treatment, Food Chemistry, 107, 753-760 (2008).

Lambert, I. y J. Kokini, Effect of L-Cysteine on the Rheological Properties of Wheat Flour, Cereal Chemistry, 78, 226-230 (2001).

Larré, C., D. Papini y Popineau, G. Deshayes, C. Desserme y J. Lefebvre, Biochemical Analysis and Rheological Properties of Gluten Modified by Transglutaminase, Cereal Chemistry, 77, 121127 (2000).

Lee, L., P. Ng, J. Whallon y J. Steffe, Relationship between Rheological Properties and Microstructural Characteristics of non-Developed, partially Developed and Developed Dough, Cereal Chemistry, 78, 447-452 (2001). 
Linko, Y. y P. Linko, Enzymes in Baking, In: Chemistry and Physics of Baking. Edited by Blanshard, J., Frazier, P. y Galliard, T. The Royal Society of Chemistry, Londres, p 113 (1986).

Lu, X. y P. Seib, Assay of Dehidroascorbic Acid in Bread and Doug Added as a Cystalline Dimer, Cereal Chemistry, 75, 200-205 (1998).

Maforimbo, E., Skurray, G.R., Nguyen, M. Evaluation of L-ascorbic acid oxidation on SH concentration in soy-wheat composite dough during resting period, LWT-Food Science and Technology, 40, 338-343 (2007).

Montgomery, D., Diseño y Análisis de Experimentos. Cap. 16. Grupo Editorial Iberoamérica. México. (1991).

Nakamura, M. y T. Kurata, Effect of L-Ascorbic Acid on the Rheological Properties of Wheat FlourWater Dough, Cereal Chemistry, 74, 647-650 (1997a).

Nakamura, M. y T. Kurata, Effect of L- Ascorbic Acid and Superoxide Anion Radical on the Rheological Properties of Wheat Flour- Water Dough, Cereal Chemistry, 74, 651-655 (1997b).

Osella C., H. Sánchez, M. de la Torre y R. González, Efecto de L-Cisteína y Proteasa sobre las Características de la Masa para Crackers, Heladería Panadería Latinoamericana, 117, 60-61 (1995).

Pyler, E., Aspect of Physical Chemistry oxidation and Reduction, in: Baking, Science and Technology I. Chicago Siebel Publishing Company, pp 269-281 (1973a).

Pyler, E., Physical and Chemical Testing Method, In: Baking Science and Technology II. Chicago Siebel Publishing Company, pp 834-859 (1973b)

Quaglia, G., Los mejorantes, In: Ciencia y tecnología de la panificación. Editoriall Acribia S.A. Zaragoza (España), pp 163-170 (1991).

Sroan, B.S., y Kaur, A. Effect of antioxidants on farinograph and amylograph characteristics of wheat flour, International Journal of Food Properties, 7, 379-391 (2004).

Sánchez, H., J. Mancuello y H. Fabre, H., Essai de Panification pour le Pain Francais, Industries des Céréales, 25, 29-32 (1983).

Tosi, E., E. Ré, R. Masciarelli, H. Sánchez, C. Osella y M. de la Torre, Whole and Deffated Hyperproteic Amaranth Flour Tested as Wheat Flour Supplementation in Mold Breads, LWT-Food Science and Technology, 35, 472-475 (2002).

Veraverbeke, W., O. Larroque, F. Békés y J. Delcour, In Vitro Polymerization of Wheat Glutenin Subunits with Inorganic Oxidizing Agents. II. Stepwise Oxidation of Low Molecular Weight Glutenin Subunits and a Mixture of High and Low Molecular Weight Glutenin Subunits, Cereal Chemistry, 77, 589-594 (2000).

Yamada, Y. y K. Preston, Effects of Individual Oxidants on Oven Rise and Bread Properties of Canadian Short Process Bread. Journal of Cereal Science, 15, 237-251 (1992).

Yamada, Y. y K. Preston, Sponge-and-Dough Bread: Effects of Oxidants on Bread and Oven Rise Properties of a Canadian Red Spring Wheat Patent Flour, Cereal Chemistry, 71, 297-300 (1994). 
